\title{
2. Rethinking Software
}

\begin{abstract}
This chapter formulates a conceptual perspective on software, starting from an attempt to situate the book in relation to existing takes on the subject. It then moves to a presentation and appropriation of Simondon's philosophy of technology, which reserves a central place to technical creation and evolution. Here, we find an understanding of technicity as a domain of life that constitutes its own substance and regularity, whilst remaining a fundamental form of human gesture. Simondon's inductive view, which frames technology as multitude of technical objects rather than idealized techne, grounds the conceptual and analytical apparatus then brought to the analysis of algorithmic techniques.
\end{abstract}

Keywords: software studies, theory of software, philosophy of technology, Gilbert Simondon

Taken together, computerization, information overload, and social diversification help us explain how algorithmic information ordering techniques have come to play such prominent roles and call attention to at least some of the social, political, and economic matters they have become entangled with. But they do not provide a clear picture of the actual technical substance that lurks behind words like 'processing', 'modulating', or 'ordering'. The rest of this book is thus dedicated to the technicities that give functional meaning to these terms, even if the shadow of their eventual application cannot be ignored. This requires an understanding of software, as it defines basic materialities and conditions of production. In this chapter, I begin to carve out my own perspective in conversation with a number of intellectual signposts, in particular the philosophy of Gilbert Simondon, which provides valuable clues for an understanding of software as historically accumulated archive of technical possibilities and of software-making as technical creation.

Rieder, B., Engines of Order: A Mechanology of Algorithmic Techniques. Amsterdam: Amsterdam University Press, 2020 DOI 10.5117/9789462986190_CHO2 


\section{Getting a Grip on Software}

Philosophy, in particular in its Anglo-Saxon analytical tradition, has long been interested in the foundations, status, and fundamental possibilities and limitations of computing (cf. Colburn, 200o; Floridi, 1999). Subjects such as the distinction between hard- and software, the ontological character of computer programs, and the specific problems computation raises for logicians have been lively areas of study since at least the 196os and 1970s. An important strand, in this context, is the discussion of the relationship between computing and the mind, famously epitomized by Dreyfus (1972) or Searle (1980), which asks whether computers could ever be capable of 'real' understanding and not just of performing clever tricks that succeed in fooling an observer. These thinkers work toward what I would call a foundational understanding of computing, which seeks to settle its ontological status in order to develop a clear, axiomatic basis that supports the deductive style of reasoning analytical philosophy favors.

Despite its very different grounding in what is often referred to as 'continental' philosophy, the field of software studies, a subfield or spin-off from media studies, often employs similar strategies. Following the broad roads opened by McLuhan (1964) and Kittler (1997a), authors like Berry (2011), Chun (2011), Hayles (2004), or Mackenzie (2006) have sought to assess the fundamental properties of computing in ways that frequently circulate around the notion of 'code' as the center of gravity. If one could clarify the specific character of this strange creature - written like text yet operating like a machine - one would be able to build all other aspects on these intellectual foundations. While the field has produced a series of attempts (e.g., Fuller, 2003; Gehl and Bell, 2012; Manovich, 2013c) to broaden the focus from computational foundations and experimentation at the margin to the mainstream of widely used software packages, volumes such as the Software Studies Lexicon (Fuller, 2008) illustrate a perspective that engages the nitty-gritty of technicity mostly through the basic building blocks of software and eschews the more compound or higher-level techniques and artifacts that populate software-making. In-depth discussion is often limited to the level of (short) code examples ${ }^{1}$ and experiments, while the broader

1 It is telling that Berry's Philosophy of Software provides a complete implementation of Quicksort (2011, p. 48) in C, in order to discuss 'code aesthetics', but says nothing about what that code actually does or what kind of intellectual substance it carries. But without capturing the technicity of the method, there is simply no means to understand why this particular example is considered 'beautiful'. Incidentally, the code snippet, taken from Oram and Wilson (2007), would be an excellent example for discussing how Simondon's notion of concretization applies to software. 
technical rationales driving the design and architecture of software artifacts and behavior remain in the background.

Using a somewhat clumsy analogy, one could say that most existing theorizations have approached software primarily through its manifestation as 'language', code, rather than as 'literature', that is, as the myriad of components and programs written in concrete settings for concrete purposes. Understanding how code operates is certainly crucial for getting a grip on software, but the actual landscape of existing objects does not follow teleologically from the mere existence of computing machinery and programmability. Similar to language, software allows for the expression of a wide range of ideas and aspirations, even if basic principles and historically sedimented trajectories of knowledge and technicity structure spaces of possibility. The basic principles of computation have indeed received considerable attention, but the vast pools of accumulated ideas, techniques, systems, or reservoirs of ready-made function have rarely been made matters of concern. Despite the many inspirations I take from strands of foundational reasoning, my goal is indeed to inquire into a small but highly significant portion of what has been expressed as software and what continues to be expressed when developers draw on available techniques to build programs.

If the notion of algorithm, which I will address in the next chapter, points toward a foundational definition of computation, the notion of algorithmic technique opens onto a vast, contingent, and heterogeneous landscape of words and things that clutter minds and solid-state drives. Because, ultimately, if our business is not with the very possibility of mechanical computation, but with software as a plethora of objects in-the-world, the question to ask becomes 'What is in an algorithm?', leading to an investigation into the methods and mechanisms that constitute and inform operation. The elegant concept of computation then quickly begins to bloat up with many different things: real computers, not just abstract Turing machines; real software, lodged in tight networks of other software, all written for a purpose; data that stand for something, outputs that have consequences. If software is indeed eating the world, then the world - culture, economics, politics, sociability, education, desire, and so forth - fills up software in return. Much like Latour's famous speed bump, we could argue that software 'is not made of matter, ultimately; it is full of engineers and chancellors and lawmakers, commingling their wills and their story lines with those of gravel, concrete, paint, and standard calculations' (Latour, 1994, p. 41). At the same time, what is the gravel of computation? computers? bits? code? How is software made and what is if made out of? Software-making can be, and certainly has been (Bowker et al., 1997), studied through a social 
scientific lens investigating work processes, social norms, situated learning, tacit knowledge, organizational structures, economic constraints, and so forth. But if there is no serious attention paid to the technical content of software, an approach limited to social or cultural embeddings risks treating technical objects as mere epiphenomena of social or cultural forces. If we consider that artifacts like Google's search ranking mechanisms have political relevance, the question what they do and how they do it necessarily involves a technical specificity that should not be ignored. An ethnographic workplace study of what used to be Amit Singhal's team ${ }^{2}$ would probably yield highly interesting insights in its own right but would be hard pressed to understand how the mechanisms at work relate to the trajectories of technicity that enable and inform their actual operation. While important parts of software-making are indeed entangled with questions of process, convention, learning, and so forth, there is a properly technical substance that sits at the center of technical practice.

The scholarly traditions mentioned above have mostly attempted to describe this substance as a series of singular principles, but the last decades have certainly seen attempts to capture and theorize subdomains and their specificities. The dominant cultural interpretations of the technical and intellectual content of software clearly come in the form of popular science books written by journalists or computer scientists, such as MacCormick's Nine Algorithms That Changed the Future or Domingos's The Master Algorithm. While these texts do an excellent job at relaying technical principles to a lay audience, their cultural analysis is mostly celebratory and based on what amounts to commonsense reasoning about society and culture. Agre's (1997a) Computation and Human Experience, which describes itself as 'a critical reconstruction of the fundamental ideas and methods of artificial intelligence research' (n.p.), remains a rare example for an attempt to produce a technically competent and theoretically informed appreciation of this particularly evocative part of computing. Works by scholars like Burrell (2016), Dourish (2017), and Mackenzie (2015, 2017a), however, have shown more recently that it is still possible to approach some of the most complex techniques in computing from a humanistic perspective with regard to their technicity and not just their social effects and entanglements. Their efforts to unpack, interpret, and critique the rationales informing actual technicities are invaluable and pioneering, raising the question why,

2 Amit Singhal is mentioned here by name because he was one of the last PhD students of a central figure in this book, Gerard Salton. Singhal left Google in 2016, allegedly over sexual misconduct, and the head of machine learning, John Giannandrea, took over the search division. 
despite the immense public interest, such works remain exceedingly rare. Mackenzie's recent Machine Learners (2017a) constitutes a particularly involved attempt to work through the technical and intellectual content of a technical subdomain that sits at the very center of current debates. Although my theoretical perspective and approach deviate in important ways, our projects share not only some of their subject matter, but also a desire to discuss and situate software as a series of particular practices. While the anxieties formulated in popular volumes such as Pariser's Filter Bubble (2011), Pasquale's The Black Box Society (2015), O'Neil's Weapons of Math Destruction (2016), or Eubanks's Automating Inequality (2018) inevitably echo through humanistic work on software, the technicities involved and their specific operational and epistemological character merit attention in their own right.

In my own attempt to investigate the substance of information ordering, I broadly follow the perspective laid out by Lev Manovich, most clearly in Software Takes Command (2013c), which develops a historically informed analysis of what he calls 'cultural software' and, in particular, of specific techniques and application packages in the domain of media creation, such as Adobe's After Effects and Photoshop. Although Manovich is certainly interested in the fundamentals of computation, his focus is squarely on what comes after computation, when software pioneers begin to turn the computer into a 'metamedium' and implement a staggering array of forms and functions into the inviting windows of graphical user interfaces. While my domain of study is a different one, I very much build on the idea that computing is marked by a continuous, cumulative, and contingent process of evolution that is in large part the result of software-making:

None of the new media authoring and editing techniques we associate with computers are simply a result of media 'being digital'. [...] 'Digital media' is a result of the gradual development and accumulation of a large number of software techniques, algorithms, data structures, and interface conventions and metaphors. (Manovich, 2013b, p. 34)

The computer may be 'undergoing continuous transformation' (Manovich, 2001, p. 64), but this transformation rests on historical processes of 'accumulation' that should not be seen as a singular stream, but as 'sedimented and layered', as 'a fold of time and materiality' (Parikka, 2012, p. 3) that produces complicated temporal patterns. This process concerns not only end-user functionality but runs through software-making itself. The constructive and cumulative 'archives' of technicity and knowledge shape 
the paths of possibility for the making of technical objects and even if they imply no teleology, they constitute the foundation for any future advancements.

The next section therefore turns to the philosophy of Gilbert Simondon to frame software as technology - not to neutralize its specificities, but to consider it as part of a fundamental mode of constructing and relating to the world, and to distill a number of conceptual and methodological resources for the analysis of concrete technical objects and their evolution. Certain distinctions, in particular between objective and objectal, between abstract and concrete, and between element, individual, and ensemble, make it possible to address algorithmic techniques for information ordering with regard to both their inner composition and their complex relational embeddings. These distinctions inform a perspective that understands the practice of software-making not primarily as 'writing code', but as the creation of technical objects, that is, of 'beings that function' (Simondon, 2017, p. 151). The recognition that technical creation is both constructive and contingent is central to this argument.

\section{Software as Technology}

Simondon's Du mode d'existence des objets techniques, ${ }^{3}$ published in 1958 and translated as On the Mode of Existence of Technical Objects (Simondon, 2017), remains to this day one of the most striking attempts to make technology a matter of philosophical concern. What makes this text a timely read 6o years after its publication is the delineation of a technical mode and substance that are distinct - although not disconnected - from other domains of being. Technology is neither understood as a manifestation of

3 All of the conceptual work in this book has been based on the French original (Simondon, 1958). While I reference the English version (Simondon, 2017) for convenience, certain translations have been amended. Most importantly, I translate the French 'la technique', used as a categorical term, with 'technology' to make a clearer distinction with 'une technique' or 'les techniques', for which I use the term 'technique'. Simondon thinks technology from the vantage point of advanced machinery and not from tools such as hammers. The English term 'technology' connotes this emphasis on 'modern' technical objects better than the specialist term 'technics' used in the recent English translation. In general, Simondon puts little emphasis on strong ontological definitions or demarcations. The term 'techne' does not appear once in Du mode d'existence des objets techniques and central conceptual distinctions, for example, between technical element, individual, and ensemble, stem from an analysis of concrete machinery. Much care is taken to not absorb these concepts into an understanding of technology that totalizes the variety of technical objects into a singular logic. 
a broad principle of thought such as instrumental rationality, nor as an outcome of social processes yielding functional responses to some needs or desires. But technology is also not framed as an external, autonomous force that stands separate from or even against human beings. On the contrary, is seen as one of the two fundamental 'phases ${ }^{4}$ - the other being religion - that characterize the mode of existence of the ensemble constituted by humans and the world after the breakdown of the original, integral mode that Simondon (2017) calls 'magical' (p. 169). Aesthetic thinking, ethics, and scientific knowledge rise from the tension between the two phases and serve to mediate between them (p. 174).

The details of Simondon's (2017) broader metaphysics are less important here than the conceptual developments that follow from them. At the outset, we find the diagnosis of a culture marked by an imbalance (p. 16) caused by the 'ignorance or resentment' (p. 15) of the central manifestation of modern technology, the machine. The popular debates on the power of 'algorithms' or artificial intelligence neatly illustrate what Simondon means: without robust technical understanding, our culture is condemned to oscillate between two contradictory attitudes: one that holds that technology is merely matter assembled to provide some utility and one that ascribes (hostile) intentionality to it (p. 17). Both attitudes reveal a technical illiteracy that locks our societies in 'alienation', keeping them from accessing the specific way of relating to the world technical creation constitutes. Even broader than its Marxist understanding, the term denotes the profound misapprehension of an entire domain of existence. While there are fundamental differences between Simondon and Heidegger, there is an element of Seinsvergessenheit ('oblivion of being') $)^{5}$ : we treat technical objects as meaningful only in relation to a use and utility, ignoring the human reality they express and constitute. Unlike esthetic objects, they are not given right of residence in the world of signification (Simondon, 2017, p. 16).

Simondon's work is thus an attempt to fundamentally rethink (contemporary) technology as a proper domain of both being and meaning. In close contact with the technical and industrial world since his childhood, he seeks to 'recover' the significance of the material world, both mediated

4 Simondon understands the concept of 'phase' not temporally but in reference to the phase difference between two curves, that is, between $f(x)=\sin (x)$ and $f(x)=\sin (x)+\pi / 2$. This means that they need to be understood in unison, even if they each has their specificity (cf. Simondon, 2017, p. 173).

5 Heidegger's (1962) critique targets the ontological reductionism manifest in attempts to narrow being to a single aspect, for example, Plato's designation of ideas as the domain of true existence. 
and constituted by technology, for human existence. Emphasizing this conceptual and normative readjustment, Chateau argues that for Simondon '[t]echnicity is an essential mode in man's relation to the world: it is an essential mode of existence for man'.

The concept of 'technicity', the term used to conceive and address a specifically technical substance, undergirds this project, putting at its center the notion of 'function'. According to Simondon, '[w] hat resides in the machines is human reality, human gesture fixed and crystallized in working structures' (Simondon, 2017, p. 18), that is, structures that perform technical operations. This quote, however, should not be read as a concession to social constructivism, where human ideas and desires that are not themselves technical in nature are imprinted onto technical objects. On the contrary, Simondon (2014) attempts to delineate a domain of 'functional meaning' (p. 28) that rests on itself, where machines signify not only through what do, but, crucially, through how they operate. There is not something properly human on the one side that is transposed or translated into technical form on the other. Technicity itself, understood as organized functioning, is an intrinsically human mode of existence and evolution (cf. Simondon, 2014, p. 321). Simondon thus joins thinkers like Leroi-Gourhan (1993), who see technology, like language, as a fundamental trait of our species. Indeed, when Simondon (2017) argues that human beings communicate by what they invent' (p. 252), he does not mean that there is some kind of message encoded in the technical object, but anticipates McLuhan's (1964) famous one-liner: the object, its technical schema and functional makeup, is the message.

To be clear, Simondon postulates neither a cybernetic equivalence between human and machine through the notion of operation nor a confluence of technical and biological evolution (cf. Guchet, 2008). Rather, distinct orders of reality enter into contact through 'transduction': individuation, of both subjects and objects, happens when preindividual realities develop relations of mutual structuration (cf. Simondon, 1989, p. 24f.). Primacy is given to the relation over its parts, but heterogeneity is not erased. Identity, so to speak, can be seen as the metastable function of a dynamic system, as long as we understand that this does not mean that all relations are equivalent or that relations emerge spontaneously or 'promiscuously' (cf. Stiegler, 1989). A technical individual is, first and foremost, characterized by strong and stable technical or causal relations between the constitutive elements that establish and maintain its functioning. These relations constitute the objective mode of existence of a technical object, in opposition to its objectal mode, which marks a detachment from its producer and 'the beginning of a 
free adventure' (Simondon, 2014, p. 27) embedded in economic, social, and psychosocial relations. ${ }^{6}$

While Simondon concedes that social factors also affect the technical object during its evolution, he insists that the objective mode is already full of meaning and value, without considering use or finality (Simondon, 2014, p. 321). His position, here, has a normative component: early manifestations of consumer capitalism's tendency to package technical objects in endless variations of the same technicities clearly do not find Simondon's approval (cf. 2014, p. 27f.). A technical object may have a 'halo of sociality' as a 'psychosocial symbol' (Simondon, 2014, p. 29), but it already - and even primarily - signifies in its objective mode and thus constitutes a domain of expression in the form of operational structures.

Using the vocabulary of Actor-Network Theory, we could say that an object's technicity realizes 'its script, its "affordance", its potential to take hold of passersby and force them to play roles in its story' (Latour, 1999, p. 177). Simondon's philosophy, however, cautions us to not move too quickly to the heterogeneous but flat assemblages Actor-Network Theory conceives. In fact, Latour's more recent An Inquiry into Modes of Existence (2013) follows Simondon in arguing that such modes delineate their own substances in ways that are more profound than a mere incommensurability between language games, because they admit other beings than words into the fold of what makes a mode specific (Latour, 2013, p. 20). Being itself is marked by difference and, as Peters (2015) claims, '[o]ntology is not flat; it is wrinkly, cloudy, and bunched' (p. 30).

When it comes to technology, this means that one should be attentive to the specificity of an object's functioning, and, on a broader level, to the question of how technology defines its own forms of evolution and transmission. The latter, in particular, is essential for understanding software and software-making as embedded in processes of accumulation and sedimentation.

\section{Technical Evolution}

For Simondon (2017), technology is characterized by a complex process of evolution. This process includes a moment of inception, an 'absolute

6 While the conceptual separation between 'objective' and 'objectal' runs through all of Simondon's work on technology, he uses the latter term only in his lecture on the psychosociology of technicity (Simondon, 2014). My more explicit opposition between the two terms follows Chateau's (2014) interpretation. 
beginning' (p. 44) of what he calls a 'schema'. He acknowledges that there is always a prehistory - my own rather meandering account of PageRank in Chapter 7 is a case in point - yet argues that the invention of the thermionic diode, for example, amounts to the creation of a new 'technical essence' that concerns, in this case, a particular way of producing asymmetric conductance. Fleming's idea to heat one of the two electrodes in a vacuum tube to assure that current can only travel in one direction then constitutes the beginning of a technical lineage that dominates electronics from the early 1900 s to the arrival of semiconductor devices in the 196os. Indeed, what constitutes the thermionic diode's essence is not merely the technical task it performs ('what it does'), since asymmetric conductance could already be produced by other means, but the specific technical principle it relies on ('how it does it'). The technical principle takes primacy since 'no fixed structure corresponds to a definite usage' (Simondon, 2017, p. 25). And, indeed, vacuum tubes acquire a whole range of initially unintended uses over time. This means that categorizations of technical objects based on use or finality cannot capture their objective dimension and that technical trajectories are not tied to single tasks but often run through many different applications and cultural segmentations. We will see this principle at work many times in the context of information ordering.

Putting the emphasis on technical schemas highlights the deep embeddedness and relationality of modern technology. Engines working with steam, combustion, or electricity, for example, may all perform the exact same work in certain conditions, but they are made out of different materials, have different sizes, weights, and operational properties, require different forms of maintenance and care, and depend on different energy infrastructures for their particular fuels. All of this means that each engine type is more compatible with certain modes of social and economic organization than with others. And these relational aspects are not static but change in conjunction with the specific evolutionary trajectories they trace over time. While it may be enough to describe an algorithm's behavior to establish its 'script' and to analyze its role in the hic et nunc of a particular setting, a deeper appreciation of computing as a technical domain has to consider schemas as 'units of becoming' (Simondon, 2017, p. 26, translation amended) that evolve and change their potentiality. Yet the way a technical trajectory unfolds is circumscribed by its technicity, even if there is no singular destination or telos (cf. Bontems, 2009). To speak with Wolfgang Ernst (2015), 'technical things form a self-referential subsystem that [...] disconnects itself from the broad "historical" time' (p. 186) and evolve according to their 'inner time' (Eigenzeit), their own temporality. 
The notion of technical essence and the process of evolution are complicated by the observation that technical 'individuals', the level of integration Simondon (2017) associates with machines (p. 77), are made of technical 'elements' such as screws, springs, or diodes, which are the most immediate carriers of technicity (p. 73ff.). Individuals, which constitute operational units and are therefore closest to the human scale (p. 77), combine and arrange elements into functioning and integrated wholes, realizing technical schemata that evolve over time. As Chabot argues, for Simondon, ' $\mathrm{t}]$ echnological invention consists of assembling a coherent system from disparate elements' (Chabot, 2013, p. 14). Technical objects are made - and increasingly so - out of other technical objects.

The distinction between element and individual also allows for a lucid conceptualization of transmission or transversality between different trajectories, since elements are more 'universal' and can be put to use in very different individuals (Simondon, 2014, p. 327). Elements developed in one context often spread to others, further underscoring the task-independence of technicity, which will be particularly important when accounting for algorithmic techniques. At the same time, Simondon (2017, p. 21) argues that the locus of technicity has steadily 'moved up', to the point where contemporary societies are organized around technical 'ensembles' that combine many individuals into coordinated systems reliant on an exchange of information rather than energy. These ensembles - laboratories, factories, industrial networks - ensure the production of both elements and individuals. The trifecta of element, individual, and ensemble allows for a nuanced analysis of technology not as techne but as sprawling ecosystems of objects and trajectories that require inductive conceptualization rather than idealistic totalization. For Simondon, technology does not exist as a singular, but as a multitude of concrete technical objects (cf. Carrozzini, 2008, p. 9).

Another reason why Simondon's approach is attractive to students of contemporary technology is the attention paid to technical evolution after the invention of a technical schema or essence. Although common in studies of technology as economic and industrial endeavor, the matter is rarely tackled in philosophical accounts. Terms like 'lineage' or 'trajectory' indeed address how a schema evolves after its basic principles have been introduced and the particular pathway it follows relates to its technical specificity (cf. Simondon, 2017, p. 26). Evolution implies moving from an abstract state, that Simondon (2017) also calls 'analytical' (p. 29) or 'intellectual' (p. 49), toward a concrete state, where the interplay between elements has become 'a system that is entirely coherent within itself and entirely unified' (p. 29). 'Concretization', then, does not denote a movement from idea to physical 
artifact but the way technical objects shift their internal composition as they iterate from one version or instance of a schema to the next. The combustion engine can serve as an instructive example: early motors were characterized by strong functional separation between parts, each element having a single task in a functional chain (p. 27). Over many cycles of refinement, motors became more integrated, synergetic, and thus concrete. Elements started to take on multiple roles, such as the cylinder block performing heat regulation to reduce material strain and to keep the electrodes of the spark plug at optimal working temperature. Instead of being largely separate steps in a chain, the elements in a concrete technical object support and sustain each other. If a technical object is a 'theater of a certain number of reciprocal causal relations' (p. 32) that assure its operational functioning, concretization is the movement toward synergy, toward an optimal state of interaction between elements.

A schema's trajectory ends when this process has reached a point of saturation and no further optimizations can be made (p. 45 f.). At that point, any improvement to task performance requires a new invention, a new essence or schema that, in turn, will begin its own march toward concretization. The switch from steam to combustion engines clearly marks such a moment. ${ }^{7}$ Trajectories are defined by this process of evolution, not by tasks performed or by companies' changing product portfolios. Contemporary machines can tie together many trajectories: in the case of computing, it is clear that the shift from vacuum tubes to semiconductors inaugurates a new technical schema for central processing units, but other crucial elements, such as storage or input and output devices define their own evolutionary pathways.

Crucial for my discussion of software is Simondon's association of the abstract state of technical objects with artisanal modes of production and the concrete state with industrial modes. The former is 'analytical', closer to the world of ideas, and more open to deviation and new possibilities (p. 29). The latter is marked by functional closure and standardization. However, it is not standardization that drives concretization; it is concretization, as convergence toward an integrated state, that stabilizes the technical object internally and thereby makes it ready for industrial production. As a consequence of this stabilization, the concrete technical object determines its uses and 'acquires the power to shape a civilization' (p. 29), while the abstract, artisanal object is shaped by external needs and desires.

7 Simondon's analysis is actually more fine-grained and puts the diesel engine, which uses compression instead of spark plugs to ignite the fuel, in its own lineage. 
However, technical evolution is not exclusively driven by internal concretization; it also involves adaptation to the geographical ${ }^{8}$ and technical milieux, for example, through better reaction to climatic conditions or a more efficient use of production capacities. The technical object sits between the natural and the technical world and, through it, each world acts on the other (Simondon, 2017, p. 56). The idea that the natural world circumscribes the fundamental possibilities of technical functioning is certainly not uncommon. Wolfgang Ernst (2015), for example, argues that technologies can only be 'discursively constructed within the confines of matter and mathematical logic' (p. 196), which means that the 'technological time proper to media stands closer to the acculturated laws of nature than to historiography and the humanities' (p. 188). In Simondon's relational ontology, however, the difference between these two worlds is pushed into the background to make room for technical evolution as a fundamentally 'constructive' process (Simondon, 2017, p. 58).

Technical invention is different from internal concretization and adaptation to the environment. Invention means that technical objects realize or actualize their own 'associated milieu', ${ }^{9}$ composed of natural and technical elements and relations that enable and sustain their operation. The creation of this third, this 'techno-geographic' milieu, points to the fundamental contingency that undergirds the evolution of technical objects: there is no teleology, no law of necessity that drives evolution, only feats of human intelligence that, in a sense, generate new materialities out of existing materialities. This is what Simondon means when he says that technical evolution is constructive: much like biological life has formed highly complex ecosystems that are populated by organisms capable of maintaining and reproducing themselves yet deeply enmeshed in various relations and interdependencies, technology has a 'fecundity' (Simondon, 2017, p. 45) to sprawl and to extend the possibilities of life itself. Terms like 'autopoiesis' or even Herbert Simon's (1996) analysis of complex systems as 'nearly decomposable' hierarchies, where every level develops properties that cannot be reduced to the level below, capture certain aspects of Simondon's

8 The term 'geographical' underscores how, for Simondon, abstract principles such as 'nature' have little interest since materiality is always a set of concrete circumstances in time and space. 9 The mixed, relational, and self-referential character of the associated milieu becomes explicit in the following quote: 'This simultaneously technical and natural milieu can be called an associated milieu. It is that through which the technical object conditions itself in its functioning. This milieu is not fabricated [fabriqué], or at least not fabricated in its totality; it is a certain regime of natural elements surrounding the technical being, linked to a certain regime of elements that constitute the technical being' (Simondon, 2017, p. 59). 
thinking here, even if they do not sufficiently account for heterogeneity and transduction. New schemas sit on top of old schemas in an endless process of constructive expansion.

While Simondon is hardly clear on this point, there may very well be a 'ground floor' of irreducible physical structures, but since human beings exist as technical beings, we inhabit a world that is always already characterized by this relational state of overflow. Simondon's (2017) rather cryptic assertion that " $[\mathrm{t}]$ he mediation between man and the world becomes itself a world, the structure of the world' (p. 193) evokes the deeply constructive nature of technology and it is essential to emphasize that in this relational ontology, the normative force of structure, that is, the capacity of what is already given to make some things possible and others not, to make some things easy and others difficult, is not seen as a confinement, but as the very condition for an endless generation of new materialities, new schemas, new (technical) essences.

Because technology itself adds to the reservoirs of technicity, it is never just an application of science, even if its possibilities are subject to the laws of nature and concretization can be seen as a way to exploit these laws for the greatest efficiency (cf. Simondon, 2017, p. 39). But it is also never just a playground of cultural imagination. An idea describing a particular use or application is not a technical idea. Gene Roddenberry thought of a 'transporter' that can 'beam' things from one place to another, but he did not invent it and, therefore, his idea is not a technical idea and the transporter not a technical object. A technical object is a being that functions and a technical idea is one that assembles an associated milieu into a potential for operational functioning. And because the reservoir of what is given does not easily bend to our will, technical invention is hard.

Simondon's perspective has been called 'unbalanced' (Mills, 2011, n.p.) for giving priority to the objective over the objectal, that is, to technicity over economic, social, and psychosocial dimensions. However, seen in the context of Simondon's broader oeuvre, which dedicates much attention to psychosocial 'transindividuation', his work on technology can be seen as a normatively charged attempt to put culture and technicity on equal footing, framing both as 'techniques for human handling' (Simondon, 2014, p. 318) that imply their own 'modes of analysis' (p. 329). Actual technical objects, once they detach from their creators, connect technicity to specific tasks in specific settings and their contribution in this specific context is thus a shared product that can be analyzed along both lines. But technology and culture are 'unbalanced' (Simondon, 2017, p. 16) because we generally do not consider technicity as a genuine domain of meaning and a viable 
avenue for the analysis of human existence. If philosophy has historically considered technology as either autonomous or defined by its use, in Simondon's perspective it is neither. It is an 'expression of life' (Simondon, 2014, p. 321) that requires a mechanology, a mode of analysis that recognizes its specificities.

\section{The Technicity of Computing}

My investigation into information ordering is largely inspired by the question of how algorithmic techniques can be understood and investigated as what Petersen (2015) calls 'functional expressions', human communication in the form of function that exercises 'an action on man' (Simondon, 2014, p. 318). This raises the question of how Simondon's perspective, formulated in the 1950s, can be productively applied to the domain of computing. We may require some adaptation and additional nuance, but his thinking can certainly inspire a course toward an analysis of technicity that moves from totalizing accounts to the universe of technical objects, sprawling and in constant evolution. My objective, here, is not to 'rectify' Simondon's account, but to continue what he set out to do, that is, to take technical objects seriously as carriers of meaning and to ask what they can tell us about the character of contemporary technology.

The distinction between element, individual, and ensemble can certainly be read as part of a metaphysics of technology, but it can also be used as a conceptual device orienting the analysis of specific technical domains. While technicity resides on all three levels, there are important differences. Elements can be seen as carriers of 'pure' or 'free' technicity (Simondon, 2017, p. 74), because they are not yet combined into systems that, so to speak, put certain demands on them. Individuals, on the other hand, are such stable systems of organized elements (p. 74) and they require 'self-regulation' (p. 73), that is, structural coherence capable of assuring their function and stability over time. As part of an individual or machine, the element is no longer an unconstrained potential, but part of a schema that defines an associated milieu and, in turn, is defined by its relationship with the regularities of existing natural and technical materialities. A good way to think about this nuance is to consider that an element has no real scenario of optimal function or failure, since it is not yet part of a context that defines what that would mean. Whether a screw fulfills its role depends on the structural weight or pressure it has to withstand when becoming part of a specific object. We cannot say that a screw is too weak without knowing what it is supposed to hold. 
When considering computers as technical individuals, one can begin by pondering hardware as composed of various kinds of elements, from voltage regulators to semiconductor parts to cables and casing. But should we treat more stable and modular units such as processors, hard drives, and screens, which are themselves made of smaller parts, also as individuals? In Simondon's perspective, they play the role of elements, since they cannot function on their own and are therefore not tied to a specific operational context or milieu that determines the specific conditions they have to perform in. But how does software fit into this equation?

Given Simondon's impressive technical awareness, it is not surprising that computers already figure prominently in his work. They are discussed as part of a larger species of 'open' machines that possess a margin of indetermination and the capacity to receive information that is then used to set actual outcomes (Simondon, 2017, p. 154). Only living beings can give themselves information, but even a mechanical piano that plays a piece according to the holes in a paper roll has the capacity to actualize a potential in different ways (cf. p. 156). While the piano already comes with built-in 'schemas of decisions' (p. 154) that define the relationship between information and action, such schemas have to be programmed into the computer to tie incoming information to particular actions (p. 154). Software is thus fundamentally part of the machine, since it defines how it functions. The hardware remains element without the software, pure technical potential that needs other elements to become a functioning whole. That also means that programming is not simply a form of adapting a machine, it is an integral part of making a machine and, consequently, a genuine domain of technical creation and invention. The reverse holds true as well: without the hardware, software is but a technical element.

This perspective resonates with Turing's fundamental take on computation (1937), where any universal (Turing) machine can simulate any arbitrary (Turing) machine, the latter specifying the actual computational procedure to be executed. But for Simondon, only a physical computer can be a technical object that functions. As technical individual it is defined both by the software procedures it executes and by its physical capabilities, such as processing speed, storage size, networking equipment, and input/output modalities. All of these elements form the associated milieu that make the individual a 'networked object' (Guchet, 2001, p. 229) that draws preexisting potential together into function. We end up with a perspective that moves away from strict ontological distinctions between hardware and software or between digital and analog and instead gives primacy to the question of how various heterogeneous elements come together to form a machine. 
This does not mean that we cannot treat software as a particular domain or type of element. What it does mean, however, is that we need to be cognizant that actual functioning is a relational outcome of elements assembled into working systems that are placed into particular objectal contexts. The constructive and relational character of technology quickly becomes evident when we look at actual objects and their trajectories.

As previously stated, a technical essence or schema has an absolute beginning. If we consider algorithms to be potential carriers of such technical schemas, we can take something like Tony Hoare's Quicksort and trace it back to roughly 1959, when Hoare was working on machine translation on visit at Moscow State University (Hoare, 2011). But Quicksort arrives at a point in time where computers not only exist, but exist at a certain stage of development, where the early programming language Autocode has been released, where other sorting algorithms have already been in use for years, and so forth. The availability of a programming language means that many of the basic elements needed to put Quicksort in place - a list-type data structure, looping functions, basic logical operators, string comparison features, etc. - were readily available and no longer had to be coded by each programmer herself in machine language. Against a somewhat naive reading of Kittler's (1997a) 'There Is No Software', where high-level programming languages represent a form of 'obscuring' or barring of access to the hardware (p. 15o), Simondon would hold that the elements of technicity these languages provide constitute a 'more': more technicity, more possibilities, new techniques to integrate and ideas to draw on, trajectories of concretization in the form of highly optimized compilers, and so forth. Construction, not confinement. There is no base layer of technicity where the 'real' operations happen, only specific instances of natural and technical potentials that are being drawn together into a technical object. And this includes hardware: while we can reason about performance characteristics of sorting algorithms in abstract terms, Hoare (2011) himself argued that Quicksort responded to the particular challenges of sequential tape storage and the small working memory available at the time.

Quicksort may have been a genuinely new schema for sorting, but its invention occurred in an environment already full of technicity that forms a reservoir or archive for technical imagination to draw on:

We can consider the technical imagination as being defined by a particular sensitivity to the technicity of elements; it is this sensitivity to technicity, that enables the discovery of possible assemblages; the inventor does not proceed ex nihilo, starting from matter that he gives form to, but from elements that are already technical. (Simondon, 2017, p. 74) 
The compositional nature of technical invention is particularly obvious when considering computing's highly layered and modular character, which I will address in more detail in the next chapter.

Crucially, elements are not dissolved when forming individuals and they constitute their own trajectories. The combustion engine forms a lineage because there are certain functional principles that run through all combustion engines and these principles are different from the those that animate the steam engine, for example. In the domain of software, the C-family of programming languages or the many Unixlike operating systems are evocative and tangible candidates for such 'units of becoming' that carry a set of functional principles through the course of an evolution. Application packages, programming libraries, or toolkits may well be pondered in similar terms as they carry schemas from one version number to the next.

When dealing with complex software artifacts like operating systems, the distinction between element, individual, and ensemble becomes unstable, but remains analytically useful. The point of view of the element points toward technicity as fundamental technical potential or force in the sense that elements have the 'capacities for producing or undergoing an effect in a determinate manner' (Simondon, 2017, p. 75). The notion of the individual addresses how these 'pure' technical forces are integrated, how an associated milieu is formed and stabilized, how continuous operation is realized. A car defines such a mutually stabilized system of almost innumerable elements and a smartphone is no different in that regard. Hardware and software are assembled in a way that each element is adjusted to the others. The application software 'fits' the processor speed, memory capacities, screen dimensions, sensor arrays, and so forth. The battery size reflects the requirements of the hardware and the charging mechanism is designed to keep it from exploding by taking into account the chassis's capacity to evacuate heat. The operating system manages the hardware resources within their limits and assures that the applications stay out of each other's memory space. As part of an individual, each element binds and is bound at the same time.

Technical ensembles, on the other hand, constitute systems that are not characterized by the creation of an associated milieu, but rather by a form of coupling that merely connects outputs to inputs, while remaining separate in terms of actual functioning. There is no search for synergetic integration; on the contrary, each individual operates in its own milieu and interactions may produce unfavorable effects (Simondon, 2017, p. 66). In an artisan's workshop, a laboratory, or a factory, phenomena like heat transfer or electromagnetic induction from one object may detrimentally affect another and shielding 
or physical separation may be necessary, even if there is 'cooperation' in terms of overall operation. The relationship between individuals in an ensemble is therefore characterized by exchange of information rather than direct technical coupling (Simondon, 2017, p. 17). Simondon compares technical elements to organs, functional units that could not subsist on their own, and individuals to living bodies (Simondon, 2017, p. 62). In this analogy, ensembles appear as 'societies', characterized by more precarious or tumultuous dynamics of relation, stabilization, and perturbation. Computer networks connecting functionally separate machines are obvious candidates for technical ensembles, but the utility of the concept is broader. Simondon argues that technical and economic value are almost completely separate on the level of the element, while individuals and ensembles connect to broader realities such as social arrangements and practices. Ensembles, in particular, point to the fact that contemporary technology functions as a series of 'industries', highlighting the deep entanglement between technologies and economies, to the point where a whole 'geology' (Parikka, 2015) of technology becomes visible: almost everything on this planet, above ground and below, has become part of networks of production and consumption. These networks are organized through flows of information that assure communication and control. ${ }^{10}$

In Simondon's work, concretization is the march from a more abstract or modular state, where elements are arranged so that every part only fulfills a single function without synergy with the others, to a state of integration where mutual adjustments yield optimal functioning. The process implies an arrangement of technicities in a way that the technical properties of an element, which may well pose problem to the functioning of the whole, are turned into a benefit. Structural integrity, weight and size, heat evacuation, favorable operational conditions, reduction in cost and resource use during both construction and operation, and even aspects such as the degree of difficulty to produce or recycle an object: these are some of the lines the process of concretization travels along. While Simondon's conceptualization of technology from the state of the art of his time is one of its greatest strengths, it also means that there is a marked focus on energy use and, in particular, on the problem of thermal buildup as the principal plague

10 Simondon engages in a substantial dialogue with cybernetics, which he generally views favorably as a means to conceptualize and manage the dynamics of ensembles, but also considers as 'insufficient', because it neither conceives technology in its entirety, nor gives adequate attention to the specific nature of technical schemas. But it is clear that he sees a systems perspective grounded in a theory of information as essential to the understanding of technical ensembles (cf. Simondon, 2017, p. 16o). 
of industrial technology. Excess heat means energy loss, impediment to function, and, in fine, structural disintegration, all causing extra costs. Although Simondon never states this directly, the concrete technical object is, first and foremost, efficient when it comes to dealing with these problems.

Computers certainly face some of the same problems 'normal' machines do, since energy efficiency and heat evacuation are two of the most pressing issues hardware engineers struggle with. Moore's law, which holds that transistor density in integrated circuits doubles roughly every two years, can be seen as a metric for concretization that indicates efficiency gains in terms of performance, energy consumption, size, and material cost per unit. While we usually venerate invention as the creation of the genuinely new, there are good reasons Simondon dedicates so much space to concretization: the emergence of modern technology as a truly pervasive force would be unthinkable without it. From a formal point of view, the first general-purpose microprocessors of the 1970s are (almost) ${ }^{11}$ identical to the chips powering today's smartphones. But steady progress in manufacturing has sped up processors by many orders of magnitude, to the point where the supercomputers in our pockets have become capable of extraordinary feats. Similar arguments can be made for storage technology, network bandwidth, or display capabilities. Concretization thus remains essential for understanding contemporary computing, even before we include the fact that the mass manufacture of computing devices raises urgent questions about energy use and waste production (cf. Parikka, 2011). But when moving to the domain of software, the notion needs to be interrogated more carefully.

Certainly, efficiency is far from irrelevant when it comes to software. But the material conditions of software introduce differences that cannot be ignored. As Reeves (1992) notes, software is cheap to build, because what he calls the 'design' of a program, its source code, is automatically transformed ('built') into machine code by a compiler - no raw materials and elbow grease are required to turn the blueprint into an artifact. But since every detail needs to be specified and programs can get extraordinarily complex, software is expensive to design. While Reeves's argument that a program's source code constitutes its design is certainly debatable, it echoes Turing's (1948) affirmation that in computing, '[t]he engineering problem of producing various machines for various jobs is replaced by the office

11 While all Turing-complete processors can calculate all computable functions, instruction sets have grown over time, often to address special-purpose logic blocks that accelerate certain calculations, for example, for video decoding, where fixed-function hardware enables higher video resolutions and compression rates at lower energy costs. 
work of "programming" the universal machine to do these jobs' (p. 4). This means that the notion of concretization requires a different understanding of efficiency. This passage on the 'imperfection' of the abstract technical object highlights where Simondon's perspective requires adaptation:

Because of its analytic character, [the abstract technical object] uses more material and requires more construction work; it is logically simpler, yet technically more complicated, because it is made up of a convergence of several complete systems. It is more fragile than the concrete technical object, because the relative isolation of each system [...] threatens, in case of its malfunction, the preservation of the other systems (Simondon, 2017, p. 30, translation amended).

If we take a program that relies heavily on preexisting modules or libraries as an example for an abstract technical object, it is true that it may require more storage space, working memory, and processing power than purposebuilt code. But the construction time aspect is completely reversed: since the compiler builds the actual technical object and reproduction comes down to mere copying, a more abstract or modular construction will be much faster and cheaper to create than a more concrete, optimized, and integrated object. Fewer lines of code are specifically written for a given program, even if the total number of lines grows.

The sharing of modular elements between projects also means that many different programs can benefit from the time invested in debugging, security, and performance tuning. Concretization in this broad sense of optimization therefore often focuses on the element, on transversal and reusable modules, packages, libraries, and frameworks, on operating systems that underpin all function, and on development tools such as programming languages and compilers that can greatly facilitate even complicated tasks such as parallelization. As I will argue in the next chapter, actual programs rely heavily on all of these things. One could go as far as to argue that much software is constructed like an ensemble in the sense that the collaborating units merely exchange information while remaining separate in most other aspects. Services like Facebook or Google Search, which rely on vast and distributed arrays of technicity, would be obvious examples, but even much more contained programs are designed around functional separation. These considerations further add to the fundamental ambiguity between element, individual, and ensemble when it comes to computing.

If software is easy to build but difficult to design, the issues coming up in design prime over those relating to material fabrication: how can code 
be organized in a way that it is easy to maintain, easy to debug or enhance, easy to reuse in other projects, or easy to transfer to other developers? How can complexity be contained or managed? In software, an abstract technical object that addresses these issues through a strategy of 'divide and conquer' (Dijkstra, 1974, p. 609) may well be more stable and 'perfect' than a concrete one. Crucially, the mass production of software is much less dependent on concretization since a less concrete program can be copied and distributed just as easily as a tightly integrated one. Even highly abstract or 'artisanal' programs may thus end up having extremely high adoption rates and contribute to a global standardization of functional patterns. This helps explain how the software landscape, seen as the sum of available software artifacts, can group around a limited number of 'superstar' applications yet host a staggering amount of variation and novelty at the same time.

Technical evolution is indeed tied to the invention of new schemata and to their concretization, but the landscape of actually existing technical objects is the result of a much wider relational process. Drawing on the same elements or technicities, different technical individuals are designed to fit different social, economic, functional, or aesthetic circumstances. Here, we find another important relationship with the particularities of technical creation. Indeed, the dimensions of abstract (or analytic) and concrete (or synthetic) connect to two modes of making, artisanal and industrial. The former implies a certain 'immaturity' in the sense that the technical object is first and foremost an object of use, necessary for daily life (Simondon, 2017, p. 103) and drowned in the particularities of a here and now that dominates the sensible universe of the craftsman (Simondon, 2017, p. 105). The latter marks a stage of 'maturity' that entails scientific rationality (p. 103) and a higher degree of detachment from the natural milieu (p. 105), resulting in the production of an industrial associated milieu where nature is largely dominated. The engineer inhabits a world of technicity that is not primarily shaped by human needs or uses, but one where 'needs mold themselves onto the industrial technical object, which in turn acquires the power to shape a civilization' (p. 29).

This characterization of the industrial mode of production in more than one way elicits Heidegger's (1998) portrayal of modern technology as Gestell, as a form of unveiling where science and mathematics 'challenge' nature with the sole purpose of extracting energy. While Heidegger promotes a return to the artisanal mode, Simondon suggests that we should pursue an outlook that incorporates both the artisanal and industrial modes and thereby achieves a relationship that is neither dominated nor dominating, but one of 'equality' (Simondon, 2017, p. 105f.). The level where this can be achieved 
most easily is that of the technical ensemble, which integrates trajectories of concretization yet remains open to the natural and social milieux. Here, Simondon proposes cybernetics as a starting point for thinking and regulating the modalities for information exchange (p. 119). But ultimately, it is the role of philosophy to introduce a mode of thinking into 'general' culture that is capable of incorporating technicity as a fundamental mode of relating to the world, thereby installing human beings as coordinators and inventors of machines: '[Man] is among the machines that operate with him' (p. 18). Simondon attributes to philosophy the role of fostering balance, homeostasis, and regulation through mediation between the theoretical and practical, the abstract and concrete, the idea and the concept, the ethical and the scientific, the technical and the religious (p. 221). To achieve this synthesis, technicity needs to be recognized as a means of human expression, which first requires a cultural sensitivity for technical objects as human creations. My emphasis on software-making is an attempt to respond to this challenge.

Software, in fact, is an area of technology where an intermingling between modes of creation is already a common phenomenon. The emphasis on abstraction and modularity on the one side and the ongoing movements of concretization on the level of tools and libraries on the other imply a constant copresence of artisanal and industrial modes. In fact, the abstract, artisanal character of software-making is strengthened by the ongoing concretization and optimization on the lower levels. The availability of robust operating systems that provide large amounts of baseline functionality, the spread of simpler and more expressive programming languages, and the proliferation of powerful code libraries for almost any purpose make complex capabilities such as ${ }_{3} \mathrm{D}$ graphics or machine learning available to almost any programmer. The underlying trajectories of concretization support a high degree of liveliness and experimentation by providing masses of elements for the assembly of individuals and ensembles. Concretization and industrialization on one level enable abstraction and artisanal production on another. One area where this becomes particularly visible is the domain of custom software written for the here and now of a particular situation. These applications are often assembled out of standardized building blocks and adapted as practices evolve. Actors working in this domain have certainly developed various strategies - from project management to automated testing - to make their production methods more industrial but, to paraphrase Reeves, software work is largely design work and therefore tends to remain abstract, intellectual, and open to context. Software, in this sense, is more immediately susceptible to objectal requirements than industrial products developed on assembly lines. 


\section{Software as Technology}

While Simondon's thinking may seem overly complicated, it proposes a rare take on the old debates between social and technical determinism that does not propose a 'middle ground' but seeks to move beyond the dichotomy altogether. Other than the distinctions between element, individual, and ensemble and between artisanal and industrial modes of technical creation, which are particularly useful for the analysis of concrete objects and settings, there are three main considerations that inform and orient my analysis of information ordering.

First, while Simondon sees technicity as a means of expression, it is not simply a surface where social forces, cultural imagination, or economic dynamics manifest to shape technical objects and their trajectories. They certainly do play crucial roles in orienting the objectal use and adaptation of technology, but technicity itself is an irreducible domain of life that constitutes the objective 'skeleton' all technical creation draws on. Technical objects have their specificity and, in Chateau's formulation, 'their technical mode of existence is not to be confused with their social or psychosocial mode of existence' (Chateau, 2014, p. 5). In line with Ernst's (2015) argument that 'radio reception is not only a cultural agreement, but a scaffolding of electromagnetism' (p. 194), the cumulatively constructed techno-geographic milieux Simondon describes are not merely projection spaces for imaginary applications or uses. It is out of the evolving space of technical potentiality that uses can emerge. The technical object becomes objectal on the basis of its technical objectivity: its psychosocial role as an object of everyday life is bounded by its technical or function capabilities. Certainly, there are questions regarding social adoption, the economics of production, and thick layers of symbolic understanding. The car has prodigious amounts of social meaning. But it only runs at the push of a pedal due to its technicity and without that capacity to propel, there would be no social role to play. To be clear, to consider the objectal mode of technical objects is not a 'mistake'. The mistake is to take the objective development of the car as a given, to take the very existence of the car as a development that needs no further explanation or interpretation beyond mentioning some technical milestones. To appreciate the radical proposal Simondon makes, we have to understand that technology is neither socially determined, nor autonomously following some internal telos. Its evolution is contingent, constructive, and relational. But it constitutes a mode of existence that is specifically technical, and it is as technicity that it constitutes an integral part of human life. The car is 'crystallized human gesture' (Simondon, 2017, p. 18) and more deeply 
'ours' than a social constructivist position would admit. The problem, for Simondon, is that the dominant culture negates and ignores the possibilities afforded by technology to express life. Technology becomes Gestell, to speak with Heidegger, not because of its intrinsic character, but because it has been banished from the realm of 'noble' expressions to the domain of mere utility. A culture seeking to find an equilibrium would have to assign to technical objects a status similar to works of art or scientific theories, while recognizing that function is their principal mode of signification.

Second, Simondon's philosophy pushes our attention toward operation rather than materiality. His thinking is certainly of materialist persuasion, but the description of technicity as the domain of function, understood as arrangement and unfolding of reciprocal causality, emphasizes notions like process, behavior, interaction, or system over more static terminology. Here, Simondon's work shows strong affinities with cybernetics (Simondon, 2017, p. 16off.). However, if cybernetics is dedicated to describing behavior in informational terms and seeks to subsume everything into a singular layer of analysis, Simondon's ontology sees relations of various kinds between entities that keep their specificities. How a particular behavior is produced makes a difference. A working system may be described as an informational process only at the price of missing the level of technicity, where materiality and function intersect. A computer is the sum of its hard- and software, the combination of various technicities that determine its capabilities and tie it to the history of technical invention. Where cybernetics abstracts away from the particularities of underlying mechanisms, Simondon moves them to the center of attention - not to engage in an exercise of ontological categorization, but to trace how different heterogeneous elements assemble into a coherent whole. Taking this perspective into the domain of computing means inquiring into the components and relationships that enable a system to do what it does, to generate its particular behavior. This is where we will encounter algorithmic techniques as instances of technicity that render particular functions possible. Following Simondon, we have to give up the search for a 'base layer' of computation and renounce modes of description that subsume everything into singular principles. Instead we marvel at all the technicity that has been brought into being, at the mass of things already 'sayable' in the medium of function, at the 'statements' have been added to the archives of technicity. More than anything, Simondon's understanding of technology installs operation as a lush domain of cumulative creation.

Third, and drawing on the two previous points, this means that there are genuinely technical forms of imagination. Creators may be driven by all kinds of motives, but the construction of a technical object or the invention 
of a new schema require technical thinking and skill. The resulting artifact constitutes a transindividual relation that 'communicates' through the function it introduces into the world and this mode of expression requires an aptness that has to be acquired. The material reality of technicity is thus mirrored by a 'mental and practical universe' that humans come to know and draw on to create technical objects (Simondon, 2017, p. 252). Only afterwards does the technical expression become part of the lived realities of individuals and societies as it detaches from its creator to begin a 'free adventure' (Simondon, 2014, p. 27) as an object of use. As I will argue in detail in the next chapter, software-makers inhabit a knowledge domain that revolves around technicity, but also includes practical and normative notions concerning application, process, evaluation, and so forth. This domain cannot be subsumed into others.

These points together constitute a view of technology that emphasizes its internal substance and richness, without negating the myriad connections it forms and upholds. Such a perspective trades foundational arguments for inquiries into constructive accumulation. The reduction of computing to mechanical computation misses the technical substances and associated knowledge spheres that allow contemporary computers to do extraordinary things, including algorithmic information ordering. To inquire into these substances and to connect them back to other cultural domains is the task Simondon set for mechanology:

In order to restore to culture the truly general character it has lost, one must be capable of reintroducing an awareness of the nature of machines, of their mutual relations and of their relations with man, and of the values implied in these relations. This awareness requires the existence of a technologist or mechanologist, alongside the psychologist and the sociologist. (Simondon, 2017, p. 19)

This book indeed develops a mechanological probe that demarcates and investigates a slice of technicity bounded by computation at the bottom and by the world of social purposes at the top. While hardly independent from these two boundaries, it constitutes a coherent domain that must neither be absorbed, à la Kittler, into the basic principle of computation, nor too readily into the organizational, political, and economic logics that dominate contemporary applications of computing. Ultimately, I hope to show that the domain of software retains a degree of openness that constitutes an opportunity for the emergence of a 'technical culture' (Simondon, 2017, p. 257). As Mercedes Bunz (2014), a keen reader of Simondon, argues, ‘[d]igitalization 
is happening, and instead of abdicating our responsibility, we must take our part more actively' (p. 54). A deeper understanding of software and software-making is one of the conditions for living up to this challenge.

\section{Bibliography}

Agre, P. E. (1997a). Computation and Human Experience. Cambridge: Cambridge University Press.

Berry, D. M. (2011). The Philosophy of Software. Basingstoke: Palgrave Macmillan. Bontems, V. (2009). Gilbert Simondon's Genetic 'Mecanology' and the Understanding of Laws of Technical Evolution. Techné: Research in Philosophy and Technology 13(1), 1-12.

Bowker, G. C., Star, S. L., Turner, W., and Gasser, L. (eds.). (1997). Social Science, Technical Systems, and Cooperative Work: Beyond the Great Divide. New York: Psychology Press.

Bunz, M. (2014). The Silent Revolution. Basingstoke: Palgrave Macmillan.

Burrell, J. (2016). How the Machine 'Thinks': Understanding Opacity in Machine Learning Algorithms. Big Data \& Society $3(1)$, 1-12.

Carrozzini, G. (2008). Technique et humanisme. Günther Anders et Gilbert Simondon. Appareil, 2, 1-12.

Chabot, P. (2013). The Philosophy of Simondon: Between Technology and Individuation (A. Krefetz, trans.). London: Bloomsbury.

Chateau, J.-Y. (2014). Présentation. In Simondon, G., Sur la technique (1953-1983) (pp. 1-21). Paris: Presses Universitaires de France.

Chun, W. H. K. (2011). Programmed Visions. Cambridge, MA: MIT Press.

Colburn, T. R. (2000). Philosophy and Computer Science. Armonk: M. E. Sharpe.

Dijkstra, E. W. (1974). Programming as a Discipline of Mathematical Nature. American Mathematical Monthly 81(6), 608-612.

Domingos, P. (2015). The Master Algorithm: How the Quest for the Ultimate Learning Machine Will Remake Our World. New York: Basic Books.

Dourish, P. (2017). The Stuff of Bits: An Essay on the Materialities of Information. Cambridge, MA: MIT Press.

Dreyfus, H. (1972). What Computers Can't Do. New York: Harper \& Row.

Ernst, W. (2015). Technophysikalische and Symbolische Medienoperationen als Herausforderung der Historischen Zeit. In S. Haas and C. Wischermann (eds.), Die Wirklichkeit der Geschichte (pp. 185-204). Stuttgart: Franz Steiner Verlag. Eubanks, V. (2018). Automating Inequality: How High-Tech Tools Profile, Police, and Punish the Poor. New York: St. Martin's Press.

Floridi, L. (1999). Philosophy and Computing: An Introduction. London: Routledge. 
Fuller, M. (2003). It Looks Like You're Writing a Letter. In M. Fuller (ed.), Behind the Blip: Essays on the Culture of Software (pp. 137-165). New York: Autonomedia. Fuller, M. (ed.). (2008). Software Studies: A Lexicon. Cambridge, MA: MIT Press.

Gehl, R. W., and Bell, S. A. (2012). Heterogeneous Software Engineering: Garmisch 1968, Microsoft Vista, and a Methodology for Software Studies. Computational Culture 2, n.p.

Guchet, X. (2001). Théorie du Lien Social, Technologie et Philosophie: Simondon Lecteur de Merleau-Ponty Les Études philosophiques 57(2), 219-237.

Guchet, X. (2008). Évolution technique et objectivité technique chez Leroi-Gourhan et Simondon. Appareil 2, 1-14.

Hayles, N. K. (2004). Print Is Flat, Code Is Deep: The Importance of Media-Specific Analysis. Poetics Today 25(1), 67-9o.

Heidegger, M. (1962). Being and Time (J. Macquarrie, \& and E. Robinson, Trans., trans.). London: SCM Press.

Heidegger, M. (1998). Traditional Language and Technological Language. Journal of Philosophical Research 23, 129-145.

Hoare, C. A. R. (2011). Interview by L. Thomas [tape recording], 8 September. Oral History of British Science, British Library. Retrieved from https:/sounds.bl.uk/ Oral-history/Science/021M-C1379X0052XX-0005Vo.

Kittler, F. A. (1997a). There Is No Software. In J. Johnston (ed.), Literature, Media, Information Systems: Essays (pp. 147-155). Amsterdam: Overseas Publishers Association.

Latour, B. (1994). On Technical Mediation. Common Knowledge 3(2), 29-64.

Latour, B. (1999). Pandora's Hope: Essays on the Reality of Science Studies. Cambridge, MA: Harvard University Press.

Latour, B. (2013). An Inquiry into Modes of Existence. Cambridge, MA: Harvard University Press.

Leroi-Gourhan, A. (1993). Gesture and Speech (A. Bostock Berger, trans.). Cambridge, MA: MIT Press.

MacCormick, J. (2011). Nine Algorithms That Changed the Future: The Ingenious Ideas That Drive Today's Computers. Princeton: Princeton University Press.

Mackenzie, A. (2006). Cutting Code: Software and Sociality. New York: Peter Lang.

Mackenzie, A. (2015). The Production of Prediction: What Does Machine Learning Want? European Journal of Cultural Studies 18(4-5), 429-445.

Mackenzie, A. (2017a). Machine Learners: Archaeology of a Data Practice. Cambridge, MA: MIT Press.

Manovich, L. (2001). The Language of New Media. Cambridge, MA: MIT Press.

Manovich, L. (2013b). Media after Software. Journal of Visual Culture 12(1), 30-37.

Manovich, L. (2013c). Software Takes Command. New York: Bloomsbury.

McLuhan, M. (1964). Understanding Media. New York: McGraw-Hill. 
Mills, S. (2011). Concrete Software: Simondon's Mechanology and the Techno-Social. Fibreculture Journal 18, n.p.

O'Neil, C. (2016). Weapons of Math Destruction: How Big Data Increases Inequality and Threatens Democracy. New York: Crown.

Oram, A., and Wilson, G. (eds.). (2007). Beautiful Code: Leading Programmers Explain How They Think. Sebastopol: O'Reilly.

Parikka, J. (2012). What Is Media Archaeology? Cambridge: Polity Press.

Parikka, J. (2015). A Geology of Media. Minneapolis: University of Minnesota Press.

Parikka, J. (ed.). (2011). Medianatures: The Materiality of Information Technology and Electronic Waste. Ann Arbor: Open Humanities Press.

Pariser, E. (2011). The Filter Bubble: What the Internet Is Hiding from You. New York: Penguin Press.

Pasquale, F. (2015). The Black Box Society. Cambridge, MA: Harvard University Press.

Peters, J. D. (2015). The Marvelous Clouds: Toward a Philosophy of Elemental Media. Chicago: University of Chicago Press.

Petersen, J. (2015). Is Code Speech? Law and the Expressivity of Machine Language. New Media \& Society 17 (3), 415-431.

Reeves, J. W. (1992). What Is Software Design? C++ Journal 2(2), 14-12.

Searle, J. (1980) Minds, Brains and Programs. Behavioral and Brain Sciences 3(3), 417-457.

Simon, H. A. (1996). The Sciences of the Artificial ( ${ }^{\text {rd }}$ ed.). Cambridge, MA: MIT Press. Simondon, G. (1958). Du mode d'existence des objects techniques. Paris: Aubier.

Simondon, G. (1989). L'Individuation psychique et collective. Paris: Aubier.

Simondon, G. (2014). Sur la technique (1953-1983). Paris: Presses Universitaires de France.

Simondon, G. (2017). On the Mode of Existence of Technical Objects (C. Malaspina and J. Rogove, trans.). Minneapolis: Univocal Publishing.

Stiegler, B. (1989). Préface. In Simondon, G., L'Individuation psychique et collective (pp. i-xvi). Paris: Aubier.

Turing, A. M. (1937). On Computable Numbers, with an Application to the Entscheidungsproblem. Proceedings of the London Mathematical Society 2(1), 230-265.

Turing, A. M. (1948). Intelligent Machinery. National Physical Laboratory Report. Retrieved from http://www.alanturing.net/intelligent_machinery/. 
